can conveniently be divided into four parts: 1, front of body; 2, back of body; 3 , front of legs ; 4, back of legs. One part is bathed at a time. Two baths a week suffice. Using a long flamed are with iron-cored carbons or a mercury vapour lamp, a 5 -minutes' exposure, $2 \mathrm{ft}$. away, suffices. Large doses result in a thickened horny layer and pigmentation which prevents the reaction of the skin. It may be as well to intermit the light bath for a month at the end of two months, and then start again. If a vita-glass screen is used, the artificial sources are made more to resemble sunlight and much safer to use. Overdosage can do more harm than good.

\title{
The Physiological Action of Ultra-Violet Radiation and its Use in the Fome.
}

\section{By W. Kerr Russell, M.D., B.S. (Dunelm).}

$\mathrm{T}$ THE founder of the science of actinotherapy was Finsen, and he published his first paper on the subject of ultra-violet radiation in 1893 . His efforts were directed to the cure of lupus (the wolf), that disfiguring disease of the skin caused by the tubercle bacillus, a disease which so often causes those attacked by it to hide themselves away in order to avoid the gaze of their fellows. This new local form of treatment devised by Finsen was a wonderful success, and it was only Finsen's early death, at the age of forty-three years, that prevented him from carrying out other most important applications of the treatment. He left at his death the complete designs for a clinic in which investigations were to be undertaken for the purpose of ascertaining more exactly what affections would lend themselves to treatment by general light baths, and among the diseases in which this treatment was to be used, he expressly mentioned tuberculosis.

Describing his proposed carbon are bath, Finsen said it consisted of a circular room in the middle of which were two gigantic arc lights of 100 amperes, suspended about six feet from the floor ; by numerous radiating partitions, bath chambers were arranged in which the patients could lie naked on couches. It was, however, only after a visit to Switzerland, where Reyn, Finsen's brother-in-law, saw the successful work of Bernhard and Rollier in the cure of extra-pulmonary tuberculosis by regulated exposure to the sun's rays, that he instituted the general light bath treatment at the Finsen Institute in 1913. Then came the War, and though it is true that hundreds of thousands of wounded men were treated by physiotherapy, and that the conflict was largely responsible for bringing physical methods of treatment into prominence, it has only been during the last few years that general ultra-violet treatment has received the recognition in Great Britain which it deserves.

Actinotherapy is still in its infancy, and a great deal of research work is necessary before the reproach of empiricism can be removed. This makes it imperative that it should only be employed with great care and discrimination. One must also remember the cruel fate which has overtaken so many of the pioneers of X-rays, and though, judging by the experience of the Finsen Institute, it is very improbable that a similar catastrophe will befall the actinotherapist, the need for the exhibition of great caution is obvious. Dosage is a very important factor in all forms of medical treatment; we make use of the beneficial action of heat when we apply a poultice to relieve the pain of inflammation, but in excess, those same heat rays can act destructively and cause a painful burn. This is the case with X-rays, where ulceration and even cancer can be caused. Ultra-violet rays, too, are harmful in excess ; the same applies to drugs, most of which, given in too great doses, can act as dangerous poisons.

It is obvious that the prevention and cure of disease should principally be the concern of those who have been specially qualified by suitable training to perform this important work. Therefore, the only persons at the present moment who should undertake ultra-violet treatment are members of the medical profession. Unfortunately, anyone can at the present time administer ultra-violet or X-ray treatment to others. It is no longer fashionable for the quack to sell pills in the market-place; he much more probably nowadays administers physical treatment in some fashionable part of the town. It has been said that there is no part of medicine which is so nearly allied to quackery as is physical treatment, and this stricture will last so long as the present chaotic state of affairs is allowed to continue. The quack, with his power to issue blatant advertisements, will continue in his present affluent state, indiscriminately maltreating suffering humanity. It all reminds one strongly of a character in Shaw's sparkling play, "The Doctor's Dilemma," whose procedure was to give his advice free, the fee for the course of treatment to be paid in advance, and cure guaranteed. A correct diagnosis is the essential 
factor in the satisfactory treatment of any form of disease.

Recently, owing to the ill-advised propaganda of certain manufacturing firms and individuals who have allowed their commercial zeal to outrun their discretion, the tendency has been rather for lay interest to exceed medical in regard to ultra-violet radiation.

There are two ways in which the ultra-violet rays can be utilised for curative purposes-exposure of the body to the sun's rays, or irradiation with an ultra-violet lamp. Ultra-violet treatment can be general, local, or internal, and in the writer's opinion the only form of ultra-violet treatment which should be used at home is general treatment, that is, exposure of the whole body to the rays.

In regard to the most suitable form of ultraviolet lamp for use in the home, the obvious choice is the carbon arc. The extreme power of the quartz mercury vapour lamp, which renders it a most valuable instrument in the doctor's hands, is a source of real danger when it is used in the home, and for the same reason, the home use of the tungsten are with its short erythema-producing dose is also undesirable. Mercury vapour lamps can, however, be purchased where either the burner is made of translucent quartz, or a screen of the same material, or of vita-glass, is placed in front of the burner; in both these cases the time of exposure is greatly lengthened, and the risks of over-dosage considerably minimised, so that no objection can be taken to the home use of lamps of this kind.

The disadvantages of the carbon are are the initial cost of a trustworthy model with automatic regulation, and the cost of the electric current necessary for the proper functioning of the lamp, which is considerable. Incidentally, several persons can be treated with one carbon arc lamp simultaneously, so that it seems that one solution of the difficulty is the formation of special clinics where ultra-violet treatment can be administered collectively at a reasonable charge under medical supervision. Special physical treatment clinics are increasing daily, especially in Germany, where there are many excellent establishments for the treatment of rheumatic disorders. However, the careful home use of ultra-violet radiation is justified by intelligent persons in good health who wish to make use of its tonic effects, but the treatment of disease is an entirely different matter, and the great temptation of a layman who has installed a lamp to treat various ailing friends and neighbours, should be firmly resisted. A doctor's advice and supervision are imperative in the treatment of disease.

The dangers of unskilled radiation treatment are very real, and many fatal cases of exfoliative dermatitis have occurred. A few years ago an accident took place in which a patient, who had been in the habit of giving himself ultra-violet treatment with a quartz mercury vapour lamp at home, while lying on his bed, fell asleep. Instead of his usual ten minutes' exposure, he was irradiated for one hour and ten minutes. Marked reddening of the skin occurred and peeling followed. Twelve days later, a general redness of the skin developed, and at the end of another five days, the heart became gravely affected and very irregular; the patient was seriously ill for four days and then gradually he began to improve. Meanwhile the peeling of the skin continued, and after the lapse of a further two weeks, a hæmorrhagic rash appeared all over the body, and afterwards the patient slowly recovered. A short while ago an acquaintance of the writer's bought a mercury vapour lamp, and his first irradiation was so ill-timed that a marked erythema developed, and as a consequence the expensive lamp was promptly laid aside.

Though the ultra-violet rays have very little power of penetrating the skin, they are nevertheless able to produce two striking peripheral effects, namely, erythema or reddening of the skin, and tanning, or pigmentation. Actinic erythema, unlike that produced by the infra-red rays, comes on after a latent period of four to twelve hours, and pigmentation follows the exythema. It must be remarked that the skin exercises an important protective function against excessive irradiation; it is also an insulating medium, an excretory and an endocrine organ, and it contains many arterioles, and is the most extensive sensory organ of the body.

There are many theories regarding the action of the ultra-violet rays. One is that some of the rays penetrate to the capillaries in the vascular layer of the skin, where they are absorbed by the blood and chemical changes occur, leading to a general body effect. Another theory is that the rays have a direct photo-electric effect on the nerve endings in the skin, and yet another that the rays act photo-chemically on the skin fat-producing vitamin $\mathrm{D}$, the bone-forming vitamin. Probably all three theories are partly true. An important action of ultra-violet rays is their power of killing bacteria. Five seconds' exposure to the rays from a mercury vapour lamp is often sufficient to kill some virulent organism. Water, which is probably the most transparent liquid to the ultra-violet rays 
that we know, can be sterilised readily by them. The sun is Nature's universal disinfectant, and it is only in darkness that the organisms which are harmful to man and other animals can flourish.

Irradiation of the body generally leads to a fall in blood pressure, and Finsen proved that there was a seasonal variation in the numbers of red blood corpuscles, the number being greatest at the end of summer and least at the beginning of spring. So that if the red blood corpuscles are reduced in number, they generally increase after exposure to the ultra-violet rays. The hæmoglobin colouring matter of the red cells also increases in amount, and this proves an analogy with plants which, when grown in darkness, are deficient in chlorophyll. Changes occur also in the white blood corpuscles; the polymorphonuclear cells decrease, and the lymphocytes and, most markedly of all, the eosinophilic cells, become more numerous. There are alterations in the blood serum, and if there is an excessive quantity of blood sugar present, it is reduced. The amount of tyrosin in the blood serum increases, but decreases when pigmentation occurs, and a basic hydrogen ion concentration generally takes place. The vasomotor reflexes of the body are stimulated by ultraviolet irradiation, and this leads to an improved regulation of the body temperature, so that a body habitually stifled by wearing an excessive amount of clothing becomes able to tolerate with comfort greater extremes of heat and cold. It is a common experience that less clothing is often worn after a course of irradiation.

Ultra-violet radiation provokes certain metabolic changes. It has already been mentioned that hyperglycæmia is reduced; fats are also oxidised, particularly by natural sun baths, but the most profound action is on the mineral metabolism, the body calcium, phosphorus, iron, and iodine being all increased in amount. The calcium salts play a very important part in the body economy. They have a tonic effect on the heart, they stimulate the peripheral circulation and decrease the permeability of the lymph and blood-vessels; in bleeding, their presence is essential before clotting can occur. Calcium salts also stimulate the phagocytes in the blood, and play an important part in repairing the local ravages of disease.

Ultra-violet rays probably influence the ductless glands through the medium of the sympathetic or vegetative nervous system. They stimulate the thyroid and sexual glands and cause depression of the suprarenal glands. It has been found experimentally that irradiation diminishes the acidity of the gastric juice. The ultra-violet rays act on the central nervous system, and cause mental stimulation. The cheering effect on our spirits of a bright sunny day has long been realised, and artificially produced ultra-violet rays generally have the same effect. Intelligence tests carried out at the Treloar Cripples' Hospital, Alton, proved that the children there were about a year in advance of crippled children of the same age living in London. Ultraviolet, like the infra-red rays, have an analgesic effect on the peripheral nervous system, thus relieving pain.

The ultra-violet lamp in the home can be installed in a bedroom, or preferably in a large spare room. It is certainly unwise to put it in the bathroom, because here the electrical dangers are very great, and many fatal cases of electrocution have been reported. Moreover, the steam rising from hot water in the bath would condense on the unlit lamp and cause certain metal parts to become rusty, and dampness damages insulation. For home use, a suspension type of lamp would probably prove most convenient; a beam should be found and a pulley securely fixed into it-the lamp should be counter-weighted so as to make it possible to raise or lower it at will. An are with cored carbons often gives off unpleasant fumes, so that the ultraviolet room should be satisfactorily ventilatedcross ceiling ventilation is the ideal, but the creation of draughts must be avoided, as the open are is very sensitive to air currents. It should be possible to heat the treatment room to a temperature of about $70^{\circ}$, as general treatment is always given to the nude body. Satisfactory wiring, able to carry the required load, must be installed, and if it is available, the direct current should be chosen. It is generally possible to obtain permission to use the ultra-violet lamp on the power circuit, and power rates are generally much cheaper than the rate for lighting. The polarity of the two terminals must be known, and a three pin, or non-reversible type of plug should always be fitted. There should also be a quick-break type of protected double pole switch to turn on and off the current. The fuses should be of the easy replacement type and some spare fuse wire should always be available. The lamp resistance can be mounted on the wall.

In my opinion the most trustworthy types of carbon arc lamp are (1) the 'short flame' arc taking 20 amperes, made at the Finsen Institute, Copenhagen, which can only be used with direct current (with 240 volts three lamps can be used in series), and (2) the 'Iong flame' are (with 240 volts two can be used in series) designed to take 30 amperes. 
The disadvantage of the short flame ares is that the maximum exposure with them reaches $2 \frac{1}{2}$ hours, whereas with the long flame arc it never exceeds an hour, and when two long flame arcs are used simultaneously, one in front and the other behind, the time needed for treatment can be cut down to half an hour. The direction of the radiant emission from these two types of lamp varies considerably. The greatest ultra-violet emission from Finsen lamps is given off at an angle of about $45^{\circ}$ to the lower carbon, whereas the maximum emission from the long flame arc is given off by the flame itself, and is therefore greatest in the horizontal direction.

With the Finsen arcs, irradiation is given while the subject is lying on a couch, first in the prone and then in the supine position. The subject should be seated on a chair during exposure to the long flame are, and first the front of the body and then the back irradiated, the are flame being on exactly the same horizontal plane as the lowermost ribs. If preferred, irradiation can also be made in the reclining position on a couch, the subject lying on his side and facing the lamp, and then lying on his other side with his back to the lamp. With carbon arc lamps, the distance of the subject from the lamp should be as short as the heat comfortably allows, but not too short, because burning with the infra-red rays can, of course, occur. The initial exposure to each side of the body with the short flame arc should not exceed ten minutes, and should be gradually increased to a total of seventy-five minutes, back and front. With the long flame are fitted with white flame carbons, an exposure of each side of the body for five minutes, at a distance of approximately four feet from the arc, should be made. This time is gradually increased to a maximum of thirty minutes.

A trustworthy alarm system should be installed to give warning at the termination of the exposure, but it is generally safer for a second person to be present.

During exposure the eyes must be carefully protected with suitable glasses or some other material which is opaque to the ultra-violet rays.

Irradiation is generally carried out every other day, and can be continued regularly for months, though it is only fair to say that many actinotherapists consider that it is advantageous to make an intermission of about a month after every twelve exposures.

\section{Ultra-Violet Radiation for Domestic Use.}

\section{By Prof. S. Russ.}

$\mathbf{I}^{\mathrm{T}}$ T seems very natural for people to want to make use of " artificial sunlight ' in their homes, especially during the rather sunless seasons, but there are some points which should be considered before it becomes a general practice, especially among those who seem to get along very well as they are.

In the first place, how nearly are artificial sources of radiation a copy of sunlight as it reaches most of us, nearly at sea-level? Perhaps the nearest approach to this is given by a high temperature filament lamp, though this will err on the side of having too big a proportion of radiation in the red and yellow part of the spectrum. Such lamps are of course largely used therapeutically, but mainly for the radiant heat which they give in a very convenient form.

All the forms of arc lamps, carbon, carbon-cored, tungsten, titanium, or mercury vapour, give out a composite radiation which is very different from sunlight, as it contains a large proportion of ultraviolet radiation. So that none of these sources which are often described as providing artificial sunlight can be said to do so unless a large part of the ultra-violet is screened off, and the remain- ing part of the spectrum very carefully compensated so as to resemble sunlight as nearly as possible.

In the second place, even if a source were invented that gave a fairly exact replica of sunlight as it comes into our houses, would it be used to any extent, and if so, for what purpose? This raises the question rather at the root of the matter ; Why this quest for body irradiation? Undoubtedly much more attention is now given to housing, particularly to ventilation and lighting; it seems in fact an inevitable part of the hygienic trend which aims at abolishing the smoke pall of big cities, and the basement life which many seem able to survive. But we shall probably not be far wrong in attributing the demand for some source of artificial sunlight rather to a wish for ultraviolet radiation, and this in turn is probably due very largely to the discovery that irradiation with ultra-violet rays can cure rickets in children. It is perhaps not so much this plain fact as the story of its unfolding that has appealed so strongly to the imagination. This discovery has shown how a deficiency disease can be cured by exposing the body to radiation which penetrates but a fraction of a millimetre into the skin. The inferences 